\title{
The Changing Demographics and Treatment of Larynx Cancer
}

\author{
Jasper Han, BS, and Baran D. Sumer, MD, FACS \\ Department of Otolaryngology, Head and Neck Surgery, Division of Head and Neck Cancer, University of Texas \\ Southwestern Medical Center, Dallas, TX
}

A dominant narrative in the treatment of larynx cancer has been balancing effective oncologic therapy with the preservation of major laryngeal functions such as breathing, swallowing, and speech. The first total laryngectomy, described by Billroth in 1873, was able to maintain the airway, and, separated the pharynx from the trachea, allowing swallowing without aspiration. ${ }^{1}$ With modern tracheoesophageal prostheses for speech, total laryngectomy provides an oncologically comprehensive operation that facilitates safe swallowing without aspiration. For this reason, total laryngectomy remains a standard of care for advanced larynx cancer and the metric by which other therapies are measured.

Despite this, the permanent tracheostoma and loss of vocal cord function has motivated interest in organ preservation since the earliest days of laryngeal surgery. Partial laryngeal surgery via a 'laryngo-fissure' or external approach was attempted as early as 1834 . The first report of this technique being employed for a malignancy was described by Buck in $1853 .^{2}$ In the 20th century, improved anesthetic techniques, postoperative care, and, above all, improved selection of cases suitable for the operation, improved cure rates, with Chevalier Jackson reporting a local control rate of $82 \%$ in $1927 .^{3}$ Further refinements such as the hemilaryngectomy reported by Gluck and colleagues ${ }^{4}$ and the supraglottic laryngectomy described by Wilfred Trotter in 1913, modified by Justo Alonso, ${ }^{5}$ and popularized by Som, Bocca, and Ogura, followed. The most significant problem solved by these trailblazing

(C) Society of Surgical Oncology 2021

First Received: 20 July 2021

Accepted: 4 August 2021;

Published Online: 24 August 2021

B. D. Sumer, MD, FACS

e-mail: Baran.Sumer@UTSouthwestern.edu surgeons was determining when partial laryngeal surgery was possible and when total laryngectomy was warranted due to anatomic and functional constraints.

Patient and tumor selection is also critical for non-surgical larynx preservation. The Veterans Affairs (VA) larynx trial demonstrated that appropriately selected patients can undergo larynx-preserving non-surgical therapy and achieve oncologic outcomes equivalent to patients treated with total laryngectomy. ${ }^{6}$ Further treatment intensification improved local control and recurrence-free survival, but was also found to lead to greater laryngeal dysfunction. ${ }^{7}$ Oftentimes, this dysfunction is subtle chronic aspiration associated with increased mortality. ${ }^{8}$ Striking the optimal balance between oncologic control and function after non-surgical therapy has become the latest frontier in larynx cancer treatment. Since the widespread adoption of chemotherapy and radiation, refinements such as intensitymodulated radiation therapy (IMRT), stereotactic body radiation therapy (SBRT), ${ }^{9}$ better pretreatment patient selection, and early laryngectomy for aspiration have improved the non-surgical treatment of larynx cancer. ${ }^{8}$ Thus, as with open partial laryngeal surgery, chemoradiation underwent refinement after initial adoption. This learning curve was proposed as a potential cause for the decrease in survival for larynx cancer observed by Hoffman et al. from 1985 to 2001, a time period bracketing the widespread adoption of non-surgical organpreservation..$^{10,11}$

Representing a comprehensive update of Hoffman's analysis, in the current issue of Annals of Surgical Oncology Li et al. show that 2- and 5-year observed survival (OS) and relative survival (RS) remained flat over the period from 2004 to 2016, a period when concurrent chemoradiation and transoral partial laryngeal surgery had already been firmly established in the armamentarium of head and neck oncologists. There was also a trend toward higher-stage disease or stage migration, with a greater 
proportion of stage IV disease seen towards the end of the study period, and with an annual increase in the risk of stage IV disease of $2.2 \%$ per year. The stage migration was driven by higher rates of $\mathrm{T} 3$ and $\mathrm{T} 4$ disease, as well as an increase in $\mathrm{N} 2$ disease.

It is unlikely this stage migration represents upstaging from expanding use of computed tomography (CT) and positron emission tomography (PET). CT was widely adopted in the 1990s for the evaluation of head and neck cancer, and the use of PET surged after Medicare approval for reimbursement in 2001. While the use of PET may have increased in the study period, as the authors point out, PET has not substantially increased detection sensitivity over CT. The rates of surgical versus non-surgical treatment also did not change during the study period, eliminating pathologic upstaging as a cause. There were also no significant shifts from laryngeal subsites such as the glottis to subsites such as the supraglottis, with greater potential for regional metastases.

Despite controlling for many indicators of underprivileged socioeconomic status, there were suggestions that underlying demographic trends may at least be partially responsible for the increase in stage IV disease. Great circle distance, defined as the miles between the patient's residence and the hospital reporting the case, was greatest for stage IV disease (11.5 miles). Stage IV patients also had the highest proportion of patients residing in counties with the lowest educational attainment and income. Notably, smoking rates strongly associated with larynx cancer were not evaluable with the National Cancer Database (NCDB). It would be interesting to analyze whether rates of smoking changed for this cohort of patients and whether pack-years were associated with stage migration. As rates of smoking have declined over the study period, this decline has not been even; rates of smoking among groups with higher socioeconomic status and education have declined precipitously, while remaining relatively high in groups that are non-White, with lower educational attainment, less income, and less access to healthcare. ${ }^{12}$

The trend toward increasing stage IV disease may thus reflect the shifting demographics of laryngeal cancer to a disease increasingly affecting underprivileged groups. Patients with lower educational attainment and socioeconomic status will have less access to early screening and advanced diagnostics. The shift of smoking prevalence towards underprivileged groups may be counteracting improvements in imaging and early detection. The continued maturation of larynx cancer treatment with better pretreatment stratification of patients and more timely use of salvage laryngectomy may have kept OS and RS from being negatively impacted, but this study highlights the important work that needs to be done in addressing disparities in care and screening for underserved populations in the US.

DISCLOSURE Baran Sumer: OncoNano Medicine Inc.: co-founder, consultant, co-inventor; Intuitive Surgical: consultant. Jasper Han has no conflicts of interest to declare.

\section{REFERENCES}

1. Stell PM. The first laryngectomy. $J$ Laryngol Otol. 1975;89:353-8.

2. Buck G. On the surgical treatment of morbid growths within the larynx. Trans Am Med Assoc. 1853;6:508-35.

3. Kirchner JA. A historical and histological view of partial laryngectomy. Bull N Y Acad Med. 1986;62:808-17.

4. Semon F, Gluck T, Tilley H, et al. A Discussion on the operative treatment of malignant diseases of the larynx. $\mathrm{Br}$ Med $\mathrm{J}$. 1903;2:1113-26.

5. Alonso JM. Conservative surgery of cancer of the larynx. Trans Am Acad Ophthalmol Otolaryngol. 1947;51:633-42.

6. Wolf GT, Fisher SG, Hong WK, et al. Induction chemotherapy plus radiation compared with surgery plus radiation in patients with advanced laryngeal cancer. $N$ Engl $J$ Med. 1991;324:1685-90.

7. Trotti A. Toxicity in head and neck cancer: a review of trends and issues. Int J Radiat Oncol Biol Phys. 2000;47:1-12.

8. Forastiere AA, Zhang Q, Weber RS, et al. Long-term results of RTOG 91-11: a comparison of three nonsurgical treatment strategies to preserve the larynx in patients with locally advanced larynx cancer. J Clin Oncol. 2013;31:845-52.

9. Sher DJ, Timmerman RD, Nedzi L, et al. Phase 1 fractional doseescalation study of equipotent stereotactic radiation therapy regimens for early-stage glottic larynx cancer. Int J Radiat Oncol Biol Phys. 2019;105:110-8.

10. Johnson J. Outcomes in laryngeal cancer: a product of the learning curve? Laryngoscope. 2006;116:1529.

11. Hoffman HT, Porter K, Karnell LH, et al. Laryngeal cancer in the United States: changes in demographics, patterns of care, and survival. Laryngoscope. 2006;116:1-13.

12. Cornelius ME, Wang TW, Jamal A, et al. Tobacco product use among adults - United States, 2019. MMWR Morb Mortal Wkly Rep. 2020;69:1736-42.

Publisher's Note Springer Nature remains neutral with regard to jurisdictional claims in published maps and institutional affiliations. 